\section{FRI0670 HOW DO GOUT-RELATED COMORBIDITIES AND LIFESTYLE FACTORS CLUSTER IN A LARGE HEALTH SURVEY OF THE GENERAL POPULATION? - RESULTS FROM THE MALMÖ PREVENTIVE PROJECT COHORT IN SOUTHERN SWEDEN}

Tahzeeb Fatima ${ }^{1}$, Peter Nilsson ${ }^{2}$, Carl Turesson ${ }^{2}$, Mats Dehlin ${ }^{1}$, Nicola Dalbeth ${ }^{3}$ Lennart T.H. Jacobsson ${ }^{1}$, Meliha C. Kapetanovic ${ }^{2} .{ }^{1}$ University of Gothenburg, Dept of Rheumatology and Inflammation Research, Gothenburg, Sweden; ${ }^{2}$ Lund University, Dept of Clinical Sciences, Malmö, Sweden; ${ }^{3}$ University of Auckland, Dept of Medicine, Auckland, New Zealand

Background: Several factors (comorbidities and lifestyle) have been shown to be associated or predict hyperuricemia or gout. Since these factors often are closely associated with each other, they may represent a few pathophysiological pathways rather than being individually important predictors. Identifying clusters of such factors may thus lead to a better understanding of the pathways involved in increased risk of gout. Two studies have previously indicated four to five phenotype clusters in prevalent cohorts of gout patients of European ancestry ${ }^{1,2}$. However, identification of clusters of gout-associated factors in the general population is lacking.

Objectives: To identify clusters of gout-related baseline comorbidities and lifestyle factors among participants in a population-based health survey. Methods: The Malmö Preventive Project is a screening program for cardiovascular risk factors, alcohol abuse and breast cancer in Malmö, Sweden. Overall, 33,346 individuals $(67 \%$ male, mean age 45.7 years at inclusion) participated. The study population was screened between 1974 and 1992. A subset of 22,057 individuals (screening period: 1975-1992) was eligible for the cluster analysis. Agglomerative hierarchical cluster analysis was performed to group similar variables and subgroup individuals with similar characteristics, using principal component and Ward's minimum variance methods in Rv3.5.2, respectively. Variables selected to cluster were obesity $\left(B M l>30 \mathrm{~kg} / \mathrm{m}^{2}\right.$ ), renal dysfunction (eGFR<60 $\mathrm{mL} / \mathrm{min} /$ $\left.1.73 \mathrm{~m}^{2}\right)$, diabetes mellitus $(\mathrm{DM})$, hypertension, prevalent cardiovascular disease (CVD), dyslipidemia (abnormal cholesterol or triglyceride values), pulmonary dysfunction $(\mathrm{PD}, \mathrm{FEV} / \mathrm{FVC}$ on spirometry $<0.7 \%)$, smoking and use of diuretics.

\section{A}

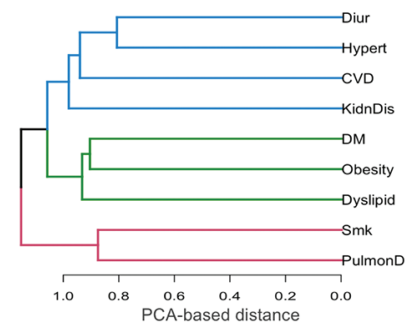

Diur: Use of Diuretics

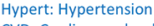

CVD: Cardiovascular disease

KidnDis: Kidney/Renal dysfunction

DM: Diabetes mellitus

Obesity: Obesity

Dyslipid: Dys/hyperlipidaemia

Smk: Smoking (current/previous)

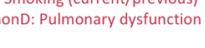

B

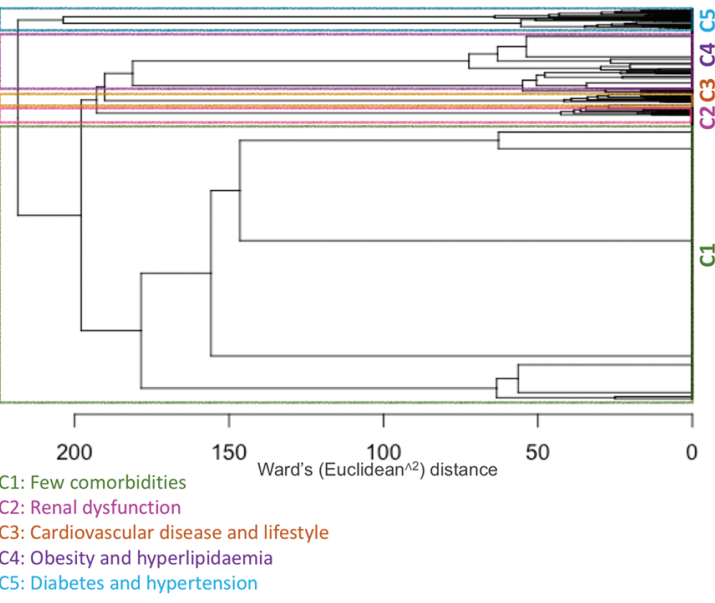

Results: Overall, $66 \%$ of the participants in the cluster analysis were males, mean age was 47 years and mean body mass index 24. Clustering of comorbidities and lifestyle factors indicated three pathways i.e. 1) mainly cardiovascular risk factors and disease, 2) variables associated with insulin resistance and 3) variables associated with PD (Fig; A).
Fig Results of cluster analysis illustrating (A) variable and (B) observation clustering

Five different clusters ( $\mathrm{C} 1$ to $\mathrm{C} 5$ ) were identified based on clustering of observations (Fig1; B). C1 $(n=16,063)$, mean age $=46$ years, characterized low rate of hypertension (14\%) and PD (15\%); none had obesity, kidney dysfunction, DM, CVD or dyslipidemia. C2 $(n=750$; mean age 51 years) had the highest proportions with gout (7.1\%) and kidney dysfunction $(100 \%)$, with no record of DM, CVD or use of diuretics. C3 ( $n=528$; mean age $=48$ years) had the highest rates of CVD (100\%) PD (22\%), smoking (74\%) and alcohol risk behaviour (41\%). C4 ( $n=3673$; mean age $=47$ years) had the highest percentage of males $(75 \%)$, the highest BMI (25.91) and the greatest proportions with obesity (34\%) and dyslipidemia (74\%), regular smoking (65\%) and alcohol risk behaviour $(36 \%)$. C5 $(n=1043$; mean age $=48$ years $)$ had by far the highest occurrence of DM $(51 \%)$, frequent use of diuretics $(52 \%)$, hypertension $(54 \%)$ and the highest percentage of abnormal liver enzyme levels (16\%).

Conclusion: Definition of clusters of comorbidities and lifestyle factors closely associated with gout, identified five separate "pathways" in this large health survey of the general population. "Pathways" relates to lifestyle, metabolism and specific comorbidity patterns. Further analyses will be performed to elucidate how these clusters predict diagnosed gout in this population.

\section{REFERENCES:}

[1] Richette P, et al. Ann Rheum Dis 2015; 74(1):142-47.

[2] Megan B, et al. Rheumatol 2018; 57(8):1358-63.

Disclosure of Interests: Tahzeeb Fatima: None declared, Peter Nilsson: None declared, Carl Turesson: None declared, Mats Dehlin: None declared, Nicola Dalbeth Grant/research support from: Amgen, AstraZeneca, Consultant for: Horizon, Hengrui, Kowa, Speakers bureau: Pfizer, Horizon, Janssen, AbbVie, Lennart T.H. Jacobsson Consultant for: LJ has received lecture and consulting fees from Pfizer, Abbvie, Novartis, Eli-Lily and Janssen, Meliha C Kapetanovic: None declared

DOI: 10.1136/annrheumdis-2019-eular.5642

\section{FRI0671 \\ SURVEY OF POTENTIAL TOXIC EXPOSURE IN PATIENTS WITH SYSTEMIC SCLEROSISIN RESCLE REGISTRY. A PRELIMINARY STUDY}

Mayka Freire ${ }^{1}$, Bernardo Sopeña ${ }^{1}$, Arturo González-Quintela ${ }^{1}$, Alfredo Guillén del Castillo ${ }^{2}$, Carles Tolosa ${ }^{3}$, Jose Antonio Vargas-Hitos ${ }^{4}$, Xabier Pla Salas ${ }^{5}$, Cristina González-Echávarri ${ }^{6}$, Antonio-J Chamorro ${ }^{7}$, Vicent Fonollosa $\mathrm{Pla}^{2}$, Carmen Pilar Simeón-Aznar ${ }^{2}$, On Behalf of Rescle Investigators, Autoimmune Diseases Study Group (Geas) ${ }^{8} .{ }^{1}$ Hospital Clínico Universitario de Santiago, Internal Medicine, Santiago de Compostela, A Coruña, Spain; ${ }^{2}$ Hospital Universitario Vall d'Hebron, Unit of Autoimmune Diseases, Department of Internal Medicine., Barcelona, Spain; ${ }^{3}$ Corporación Sanitaria Universitaria Parc Taulí, Department of Internal Medicine, Sabadell, Barcelona, Spain; ${ }^{4}$ Hospital Universitario Virgen de las Nieves, Department of Internal Medicine, Granada, Spain; ${ }^{5}$ Consorci Hospitalari de Vic, Unit of Systemic Autoimmune Diseases, Department of Internal Medicine., Vic, Barcelona, Spain; ${ }^{6}$ Hospital Universitario de Cruces, Autoimmune Diseases Research Unit, Department of Internal Medicine., Barajaldo, Spain; ${ }^{7}$ Complejo Asistencial Universitario de Salamanca, Internal Medicine, Salamanca, Spain; ${ }^{8}$ GEAS, Madrud, Spain

Background: Systemic sclerosis (SSc) is a systemic autoimmune disease with extremely heterogeneous clinical features and unknown etiology, although numerous studies suggest a relationship with environmental and occupational factors. So far there is little information on whether toxic substances can play a relevant role in its phenotypic expression (1)

Objectives: To analyze in a cohort of patients with SSc the proportion of patients exposed to toxic and their correlation with epidemiologic, clinical and serological data.

Methods: A survey was conducted aimed at the knowledge of the working life of patients from six centers belonging to the Spanish Scleroderma Registry (RESCLE), categorizing them in six groups: no potential exposure to toxic substances, potential exposure to silica, to hydrocarbons, to organic solvents, to mixed toxics (silica and/or hydrocarbons and/or organic solvents) and to another toxics. In all patients 87 epidemiological, clinical and analytical variables included in the registry were analyzed, carrying out a comparative study between groups.

Results: 225 SSc patients were selected. Of these, 81 patients $(36 \%)$ had worked in professions with potential risk of toxic exposure, 64 women out of the 227 included $(28 \%)$ and 17 men out of the 28 included $(60 \%)$. The toxic agent most frequently involved was silica in 29 patients $(35.8 \%)$, followed by hydrocarbons in $21(25.9 \%)$, mixture of toxic 